\title{
Diferenças no processamento cerebral, através do ritmo gama, durante o pensamento divergente
}

\author{
Brain processing differences through gamma rhythm during divergent
}

\author{
Ana Amancio Santos da Silva ${ }^{1}$, Euclides Mauricio Trindade Filho ${ }^{2}$
}

\begin{abstract}
RESUMO
Objetivo. Comparar o mapeamento topográfico cerebral durante o pensamento divergente. Método. A amostra foi formada por 11 voluntários de exatas e 11 voluntários de humanas, com idade entre 18 e 40 anos. Os participantes foram submetidos a testes de pensamento divergente, sendo projetados através de data show e registro do eletroencefalograma durante a leitura. Resultados. Durante a atividade de invenção de nomes, houve o predomínio do ritmo gama no grupo de humanas, nas áreas anterior esquerda, anterior direita e na área posterior esquerda. Já na atividade de uso alternativo houve o predomínio do ritmo gama no grupo de exatas na área posterior esquerda. Já na atividade de matemática ocorreu um maior processamento de atividade mental na área anterior esquerda em ambos os grupos. Também foi observado um maior processamento cerebral na área posterior direita no grupo de exatas. O grupo de humanas utilizaram mais á área anterior direita durante a atividade de invenção de nomes, enquanto que o grupo de exatas utilizaram mais a área posterior esquerda, isso evidenciado na atividade de uso alternativo de objetos. Conclusáo. Mostrando a associaçáo do ritmo gama com os estados de maior ativação do córtex cerebral durante a programação e execução de atividades cognitivas.
\end{abstract}

Unitermos. Eletroencefalograma, Mapeamento Encefálico, Cérebro

Citaçáo. Silva AAS, Trindade-Filho EM. Diferenças no processamento cerebral, através do ritmo gama, durante o pensamento divergente.

\begin{abstract}
Objective. To compare the cerebral topographic mapping during divergent thinking. Method. The sample consisted of 11 volunteers and 11 volunteers accurate human, aged between 18 and 40 years. The participants underwent tests of divergent thinking, being designed by date show and electroencephalographic recording during playback. Results. During the activity of inventing names, there was predominance of rhythm in range of human group in the left anterior areas, right front and left rear area. Already in the activity of the alternative use of rhythm predominated in the group of exact range in the left rear area. Since the activity was higher mathematical processing of mental activity in the left anterior area in both groups. There was also an increased brain processing in the right rear area of the spot group. The group used human will over right anterior area during the activity of inventing names, while the group used more accurate the left rear area, this evidenced in the activity of the alternative use of objects. Conclusion. Showing the combination of rhythm with the range states of greater activation of the cerebral cortex during the planning and execution of cognitive activities association gamma of rhythm with the range states of greater activation of the cerebral cortex during the programming and implementation of cognitive activities.
\end{abstract}

Keywords. EEG, Brain Mapping, Brain

Citation. Silva AAS, Trindade-Filho EM. Brain processing differences through gamma rhythm during divergent.
Trabalho realizado na Faculdade Estácio de Alagoas e na Universidade Estadual de Alagoas (UNCISAL). Maceió-AL, Brasil.
Endereço para correspondência: UNCISAL, Núcleo de Ciências Fisiológicas Av. Siqueira Campos, 2095. Trapiche CEP 57000-000, Maceió-AL, Brasil. e-mail: emtfilho@gmail.com

Pesquisa financiada pelo PIBIC/CNPq. 


\section{INTRODUÇÃO}

Ao nascer, todo ser humano possui centenas de bilhôes de neurônios, mas os mesmos sofrem pequena redução ao longo da vida. Nos dois primeiros anos crescem, sobretudo os apêndices mediante os quais cada célula nervosa envia sinais a milhares de outras. Pontos especiais de contato, as sinapses transmitem as informaçóes entre as diferentes células. Por intermédio de uma quantidade superior a centenas de trilhôes dessas ligaçóes sinápticas, os neurônios se reúnem em redes capazes de se comunicar entre si, mesmo a distância. De início, surgem sinapses em profusão, uniformemente distribuídas. Quando, porém, certos neurônios respondem a estímulos que frequentemente se manifestam em conjunto, disparando com igual freqüência de forma sincronizada, as sinapses entre tais neurônios se fortalecem e perduram por bastante tempo. Assim, os processos de aprendizagem modelam o cérebro, dissolvendo conexôes pouco utilizadas ou fortalecendo as ativas de uso freqüente. Então o desenvolvimento das capacidades cognitivas e o do cérebro estão vinculados um ao outro de forma indissociável ${ }^{1}$.

As funçóes cognitivas vêem sendo analisadas nos últimos anos, através dos exames de imagens: ressonância magnética funcional (RMF) e tomografia com emissão de pósitron (TEP). Estas duas técnicas têm excelente poder de resolução espacial, uma vez que, correlaciona de forma precisa a atividade funcional com a área anatômica geradora. Outra vantagem desses dois exames de imagens é o seu poder anatômico tridimensional, isto é, tanto as áreas corticais quanto as estruturas subcorticais podem ser funcionalmente identificadas. No entanto estas duas técnicas têm uma resolução temporal pobre. Os fenômenos cognitivos podem acontecer na escala de milisegundos, enquanto que as correlaçóes obtidas pela RMF e TEP exigem uma janela de segundos ${ }^{2}$. Por outro lado o eletroencefalograma (EEG) embora tenha uma resolução espacial limitado às áreas corticais, o registro da atividade elétrica cerebral em tempo real permite uma excelente resolução temporal.

As variaçóes de freqüência do EEG são agrupadas em cinco categorias; alfa, beta, teta delta e gama. A banda delta contém freqüências abaixo de $4 \mathrm{~Hz}$ e, normalmente, é observada nos estágios mais profundos do sono. Quando presente em indivíduos em vigília sempre indica anormalidade ${ }^{3}$. A banda teta representa variaçóes de freqüência entre 4 e $8 \mathrm{~Hz}$. É normalmente encontrado durante o sono. $\mathrm{O}$ ritmo teta pode ser encontrado normalmente na infância podendo ser utilizado como um elemento para estudar a maturação do sistema nervoso ${ }^{4}$. O ritmo alfa designa a presença de flutuaçóes de freqüência na faixa de 8 a 13Hz. É observado facilmente quando o indivíduo fecha os olhos, encontra-se relaxado e com relativa inatividade mental ${ }^{4,5}$. No ritmo beta as freqüências do EEG estão acima de $13 \mathrm{~Hz}$, e geralmente não excedem os $35 \mathrm{~Hz}$. A atividade beta é mais freqüente nas regióes de maior suprimento sangüíneo e, portanto está correlacionada com o nível de atividade cognitiva particularmente com a consciência ${ }^{6}$. Mais recentemente, alguns autores têm sugerido que freqüências entre 25 a $45 \mathrm{~Hz}$, que eles denominaram de ritmo gama, representaram correlatos elétricos da execução de atividades cognitivas. Assim o registro do ritmo numa determinada área cortical durante a execução de uma atividade mental sugeriria que essa área cortical seria a responsável pelo processamento da atividade mental.

O psicólogo americano Joy Paul Guilford (18971987) desenvolveu no final dos anos 40 um modelo de entendimento humano que serviu de fundamento à pesquisa moderna sobre a criatividade. $\mathrm{O}$ ponto decisivo na concepção de Guilford foi a distinção entre pensamento convergente e divergente. O pensamento convergente visa diretamente a uma única possibilidade correta de solução para determinado problema. Já o divergente seria o raciocínio criativo. Segundo Guilford, em primeira linha os testes de QI exigem pensamento convergente. Afinal sempre se trata de procurar, com auxílio da lógica, uma solução ortodoxa que se possa classificar como certa ou errada, de modo claro. No entanto, pessoas criativas destacam-se, sobretudo porque seu intelecto, ao confrontar-se com um problema, supera os esquemas mentais já arraigados e trilha novos caminhos. Guilford definia a criatividade justamente como a capacidade de "encontrar respostas inusitadas, às quais se chega por associaçóes muito amplas". E aqui entra em cena o pensamento divergente, com a finalidade de produzir diversas soluções possíveis. "No pensamento divergente avança-se para muitos lados, tâo logo seja necessário, ele muda de direção e leva com isso a uma pluralidade de respostas que 
podem ser todas elas, corretas e adequadas", explicou em $1950^{7,8}$.

Deste modo, esta pesquisa teve como objetivo analisar o mapeamento cerebral e as diferenças no processamento cerebral nos indivíduos da área de humanas e exatas através da motivação ao pensamento divergente.

\section{MÉTODO}

\section{Amostra}

Após a aprovação do protocolo de pesquisa pelo Comitê de Ética da Faculdade Estácio de Alagoas 201006-026/3672. Foram estudados 22 voluntários (11 voluntários de exatas e 11 voluntários de humanas). Esses voluntários foram recrutados pelos pesquisadores entre profissionais e estudantes, dos últimos períodos dos cursos universitários, das áreas de humanas e exatas. A pesquisa foi realizada no laboratório de fisiologia e farmacologia da Estácio, Faculdade de Alagoas (FAL) e no laboratório de fisiologia da UNCISAL (Universidade Estadual de Ciências da Saúde de Alagoas), Maceió-AL. Foram excluídos os indivíduos que embora tenham formaçôes universitárias das áreas de humanas ou exatas não exerçam atividades congruentes com a sua formação, que estejam fazendo uso de drogas psicotrópicas ou que apresentem alteraçóes comportamentais.

\section{Procedimento}

Utilizamos para a realização do registro eletroencefalográfico, o eletroencefalógrafo da marca EMSA, modelo 2031, de 20 canais, processador de 12bits e frequência amostral de 256 pontos. A colocação dos eletrodos de escalpo, obedeceu ao sistema internacional 10-20, com 22 eletrodos (Fp1/Fp2, F7/F8, T3/T4, T5/T6, F3/ F4, C3/C4, P3/P4, O1/O2, FZ,CZ, PZ e OZ, A1/ A2) conforme preconizado pela Sociedade Brasileira de Neurofisiologia Clínica e pela American EEG Society ${ }^{9,10}$. Cuja variável qualitativa nominal foi à formação acadêmica, sendo caracterizado como: humanas ou exatas. E a variável secundária de análise topográfica da frequência cerebral, sendo de forma quantitativa foi medida pelo percentual dos ritmos gama em cada quadrante cerebral.

Os exames eletroencefalográficos foram realizados sempre no período matutino entre 8:00 e 12:00h.
Cada sessão consistiu, inicialmente, de dez minutos de registro basal em vigília. Em seguida o registro eletroencefalográfico foi continuado simultaneamente com: Tarefa de Uso Alternativo (atividade 2): nesta tarefa era apresentado um objeto na tela do computador e o participante teria que pensar em um uso alternativo, não usual, para aquele objeto (CADEADO). Tarefa de Características do Objeto (atividade 3): nesta tarefa o participante teria que pensar nas características e uso comum no dia a dia do objeto apresentado (FACA).Tarefa de Invençáo de Nomes (atividade 1): era apresentada uma abreviatura formada por duas letras e o participante teria que pensar em palavras que poderiam representar as letras da abreviatura (FM).Tarefa de Matemática (atividade 4): era apresentado um problema matemático simples e o participante teria que pensar na resolução do mesmo. Tarefa de Complementaçáo de Prefixos (atividade 5): era apresentado um prefixo e o participante teria que pensar no maior número de palavras que completem adequadamente o prefixo (PRE).

\section{Análise Estatística}

Foram aplicadas técnicas de estatística descritiva, incluindo tabelas e gráficos. Foram utilizados também o modelo estatístico BioEstat 5.0, teste t para amostras pareadas, teste não paramétrico Mann-Witney para amostras independentes. Um nível de significância de 0,05 foi aceito.

\section{RESULTADOS}

A análise dos dados obtidos durante o repouso mostrou predomínio do ritmo gama nos indivíduos da área de humanas nas regióes anterior esquerda $(\mathrm{p}=0,04)$ anterior direita $(\mathrm{p}=0,03)$ e na área posterior esquerda $(\mathrm{p}=0,004)$. Por outro lado foi observado um predomínio do ritmo gama nos indivíduos de exatas na região posterior direita ( $\mathrm{p}=0,009$; Tabela 1$)$.

A análise dos dados obtidos durante a atividade 1 , entre os grupos de humanas e exatas mostrou predomínio do ritmo gama nos indivíduos de humanas nas áreas anterior esquerda $(p=0,03)$, anterior direita $(p=0,005)$ e na área posterior esquerda ( $\mathrm{p}=0,05$; Tabela 2$)$.

A análise dos dados obtidos durante a atividade 
Tabela 1. Média e desvio padrão (DP) dos valores de ritmo gama, nos indivíduos de humanas e exatas, durante o repouso, nas regióes anterior esquerda $(\mathrm{AE})$, anterior direita $(\mathrm{AD})$, posterior esquerda (PE) e posterior direita (PD).

\begin{tabular}{ccccccccc}
\hline & \multicolumn{4}{c}{ Humanas } & \multicolumn{4}{c}{ Exatas } \\
\hline Área & AE & AD & PE & PD & AE & AD & PE & PD \\
\hline média & $27,4^{*}$ & 27,6 & 27,0 & 27,0 & 26,85 & 26,98 & 26,61 & $27,65^{*}$ \\
DP & 1,5 & 1,4 & 1,1 & 0,9 & 1,06 & 0,82 & 0,66 & 1,25 \\
\hline $\mathrm{p}<0,05$. & & & & & & & &
\end{tabular}

2, entre os grupos de humanas e exatas mostrou predomínio do ritmo gama nos indivíduos da área de exatas na região posterior esquerda $(\mathrm{p}=0,04$; Tabela 3$)$.

A análise dos dados obtidos durante a atividade 3 , entre os grupos de humanas e exatas não mostrou predomínio do ritmo gama nos indivíduos da regiáo de humanas e exatas.

A análise dos dados obtidos durante a atividade 4 , entre os grupos de humanas e exatas mostrou predomínio do ritmo gama nos indivíduos da área de humanas na região anterior esquerda $(\mathrm{p}=0,006)$. Por outro lado foi observado um aumento do ritmo gama indivíduos de exatas na regiáo posterior direita ( $\mathrm{p}=0,007$; Tabela 4).

A análise dos dados obtidos durante a atividade 5, entre os grupos de humanas e exatas mostrou predomínio do ritmo gama nos indivíduos da área de humanas nas região anterior esquerda $(\mathrm{p}=0,00006)$, região anterior direita $(\mathrm{p}=0,001)$, regiáo posterior esquerda $(\mathrm{p}=0,003)$. Por outro lado foi observado um predomínio do ritmo gama nos indivíduos de exatas, na regiâo posterior direita $(\mathrm{p}=0,01$; Tabela 5).

\section{DISCUSSÃo}

Os resultados obtidos em nosso estudo sobre os ritmos: Teta, Alfa, beta e Gama confirmaram os encontrados na literatura quanto serem relacionadas às mudanças na atividade cognitiva, no aprendizado de tarefas cognitivas assim como no aprendizado de tarefas motoras ${ }^{11}$.

Estes dados corroboram com o que é descrito na literatura sobre o comportamento de ritmos eletroencefalográficos na aprendizagem cognitiva e motora ${ }^{11,12}$. De forma simplificada, Alfa está descrita como a atividade representativa de estados cognitivos relacionados a estados de vigília; e Gama, presentes em condiçôes de planejamento de movimentos. Além disso, Teta e Gama são relacionadas com evocação e memórias motoras. Foram apresentadas evidências cognitivas de aprendizagem motora usando o eletroencefalograma (EEG) cujo protocolo e tarefa buscou motivar dois violonistas a treinar e aprender a tocar uma seqüência de notas oferecidas em partitura e áudio. No treinamento para memorização da tarefa, a banda de freqüência Alfa comportou-se em freqüências maiores comparados com o estado de base, da mesma forma em que Teta na etapa de audição, confrontadas com a leitura (rosa). Alteraram-se de formas diferentes no tempo, principalmente em frequências Teta e Gama, sendo o efeito estatístico das etapas sobre Teta predominante ${ }^{11,13}$.

Em outro estudo também por meio de EEG, realizado com homens e mulheres, que teve objetivo de investigar a atividade cerebral durante a resolução criativa de problemas, foi observado que a geração de idéias originais foi associada com a sincronização de alfa em regiōes frontais do cérebro e com um padrão difuso e generalizado de sincronização alfa em regióes parietais corticais ${ }^{14}$.

Há evidências em favor da interpretação que o aumento do ritmo gama entre diferentes áreas do córtex podem manifestar-se através de uma cooperação mais estreita destas regióes no processamento cognitivo ${ }^{13}$. A nossa pesquisa foi projetada no intuito de investigar possível probabilidade de pessoas dos grupos de humanas e exatas usarem áreas distintas do córtex cerebral. Nós medimos a atividade do cérebro por meio de EEG. Durante o repouso os indivíduos do grupo de humanas e exatas, passaram dois minutos de registro basal em vigília, com olhos fechados. Conforme análise estatística houve diferenças do ritmo gama nas áreas anterior esquerda, anterior direita e posterior esquerda nos indivíduos do grupo de humanas, deste modo essas áreas corticais tiveram uma maior processamento motor. Por outro lado durante o repouso nos

Tabela 2. Média e desvio padrão (DP) dos valores de ritmo gama, nos indivíduos de humanas e exatas, durante a execução da atividade 1, nas regióes anterior esquerda $(\mathrm{AE})$, anterior direita $(\mathrm{AD})$, posterior esquerda (PE) e posterior direita (PD).

\begin{tabular}{ccccccccc}
\hline \multicolumn{4}{c}{ Humanas } & \multicolumn{4}{c}{ Exatas } \\
\hline Área & AE & AD & PE & PD & AE & AD & PE & PD \\
\hline média & $26,9^{*}$ & $26,7^{*}$ & $27,3^{*}$ & 26,4 & 26,79 & 26,44 & 27,17 & 27,17 \\
DP & 1,2 & 0,8 & 0,9 & 0,4 & 0,93 & 0,44 & 1,18 & 1,34 \\
\hline p<0,05. & & & & & & & &
\end{tabular}


Tabela 3. Média e desvio padráo (DP) dos valores de ritmo gama, nos indivíduos de humanas e exatas, durante a execução da atividade 2, nas regióes anterior esquerda $(\mathrm{AE})$, anterior direita $(\mathrm{AD})$, posterior esquerda (PE) e posterior direita (PD).

\begin{tabular}{ccccccccc}
\hline \multicolumn{1}{c}{ Humanas } & \multicolumn{4}{c}{ Exatas } \\
\hline Área & AE & AD & PE & PD & AE & AD & PE & PD \\
\hline média & 26,4 & 26,5 & 26,6 & 27,1 & 26,82 & 26,95 & $26,86^{*}$ & 27,16 \\
DP & 0,3 & 0,5 & 0,5 & 1,1 & 0,95 & 0,77 & 1,02 & 1,08 \\
\hline $\mathrm{p}<0,05$. & & & & & & & &
\end{tabular}

indivíduos do grupo de exatas ocorreu um aumento do ritmo gama na área posterior direita. Deste modo, podemos ratificar a existência de diferenças no processamento cerebral durante o repouso entre os indivíduos dos grupos de humanas e exatas. Durante a atividade 1 de invenção de nomes, houve nos indivíduos do grupo de humanas, o predomínio do ritmo gama, de modo relevante, nas áreas anterior esquerda, anterior direita e na área posterior esquerda. Já na atividade 2 de uso alternativo, houve nos indivíduos do grupo de exatas, o predomínio do ritmo gama, de modo relevante na área posterior esquerda. Durante a atividade 3 de caracterização de objeto, não houve diferenças no processamento cerebral nos indivíduos dos grupos estudados. Já na atividade 4 de matemática, houve nos indivíduos do grupo de humanas e exatas, um maior processamento de atividade mental na área anterior esquerda. Por outro lado foi observado um maior processamento cerebral na área posterior direita dos indivíduos do grupo de exatas. Durante a atividade 5 de complementação de prefixos, houve nos indivíduos dos grupos de humanas, predomínio do ritmo gama, de modo relevante nas áreas anterior esquerda, área anterior direita, área posterior esquerda. Por outro lado foi observado um maior processamento motor na área posterior direita dos indivíduos do grupo de exatas. Todas essas tarefas foram cons-

Tabela 4. Média e desvio padrão (DP) dos valores de ritmo gama, nos indivíduos de humanas e exatas, durante a execução da atividade 4, nas regióes anterior esquerda $(\mathrm{AE})$, anterior direita $(\mathrm{AD})$, posterior esquerda (PE) e posterior direita (PD).

\begin{tabular}{ccccccccc}
\hline \multicolumn{1}{c}{ Humanas } & \multicolumn{4}{c}{ Exatas } \\
\hline Área & AE & AD & PE & PD & AE & AD & PE & PD \\
\hline média & 26,6 & 26,5 & $26,5^{*}$ & 26,4 & 26,65 & 26,82 & 26,65 & $27,21^{*}$ \\
DP & 1,1 & 0,6 & 0,4 & 0,3 & 1,07 & 0,95 & 0,82 & 1,31 \\
\hline p $<0,05$. & & & & & & & &
\end{tabular}

truídas para permitir neurofisiológicamente um poderoso contraste entre as tarefas experimentais e assim motivar o pensamento divergente.

\section{CONCLUSÃO}

Os dados obtidos com essa pesquisa confirmam os estudos sobre o ritmo gama, mostrando a sua associação com os estados de maior ativação do córtex cerebral durante a programação e execução de atividades cognitivas.

Nas atividades de invenção de nomes os indivíduos das áreas de humanas utilizaram mais áreas corticais do que os indivíduos da área de exatas. $\mathrm{Na}$ atividade de uso alternativo os indivíduos da área de exatas utilizaram mais intensamente a área posterior esquerda. $\mathrm{Na}$ atividade de matemática em ambos os grupos utilizou-se mais intensamente a área anterior esquerda.

Tabela 5. Média e desvio padrão (DP) dos valores de ritmo gama, nos indivíduos de humanas e exatas, durante a execução da atividade 5, nas regióes anterior esquerda $(\mathrm{AE})$, anterior direita $(\mathrm{AD})$, posterior esquerda (PE) e posterior direita (PD).

\begin{tabular}{ccccccccc}
\hline \multicolumn{1}{c}{ Humanas } & \multicolumn{4}{c}{ Exatas } \\
\hline Área & AE & AD & PE & PD & AE & AD & PE & PD \\
\hline média & $26,8^{*}$ & 27,0 & $27,2^{*}$ & 27,0 & 26,37 & 26,63 & 26,58 & $26,77^{*}$ \\
DP & 0,9 & 1,1 & 1,0 & 0,7 & 0,35 & 1,04 & 0,76 & 0,53 \\
\hline p $<0,05$. & & & & & & & &
\end{tabular}

\section{REFERÊNCIAS}

1.Friedrich Gerhard, Preiss Gerhard. Educar com a cabeça: Ao aprendermos, nossas conexões cerebrais se modificam. Com o apoio da neurodidática, neurocientistas poderão ajudar professores e pedagogos a desenvolver novas estratégias de ensino e aprendizado. Rev Viver Mente Cereb 2006;157:50-7.

2.Braga NIO. A importância do mapeamento topográfico em neurologia. In: Nitrini R, Machado LR. Condutas em neurologia 1993. São Paulo: Clínica Neurológica HC/FMUSP, 1993, p.9-14.

3.Duffy LA. Mapeamento Topográfico Cerebral. São Paulo: Ateneu, 2002.

4.Niedermeyer E. Dipole theory and electroencephalography. Clin Electroenceph 1996;27:121-31.

5.Daly DD, Pedley TA. Current Practice of clinical electroencephalography. Philadelphia: Lppincott-Raven, 1997, p.831-48.

6.Ingvar M. Pain and functional imaging. Philos Trans R Soc Lond B Biol Sci 1999;354:1347-58. http://dx.doi.org/ 10.1098/rstb.1999.0483

7.Guilford JP. Creativity. Am Psychol 1950;5:444-54.

8.Benson DF. The neurology of thinking. New York: Oxford University Press, 1994, p.2308-14. 
9.American Electroencephalographic Society. Guideline fourteen: guidelines for recording clinical EEG on digital media. J Clin Neurophysiol 1994;11:1145.

10.Luccas FJC, Braga NIO, Fonseca LC, Frochtengarten ML. Recomendaçôes para o registro e interpretação do mapeamento topográfico do eletrencefalograma (EEG) e potenciais evocados sensoriais (PES): Parte I: aspectos gerais. Braz J Epilep Clin Neurophysiol 1996;2:175-82.

11.Bonini-Rocha AC, Chiaramonte M, Zaro MA, Timm MI, Wolff D. Observação das evidências cognitivas de aprendizagem motora no desempenho de jovens violonistas monitoradas por eletroencefalograma: um estudo piloto. Cienc Cogn 2009;14:103-20.

12.Braga NIO. Análise quantitativa do eletrencefalograma: aspectos e aplicaçôes clínicas. Rev Neurocienc 1997;5:14-9.

13.Brust JCM. A Prática da neurociência: das sinapses aos sintomas. Rio de Janeiro: Reichmamm \& Affonso, 2000, 300p.

14.Fink A, Grabner RH, Benedek M, Reishofer G, Hauswirth V, Fally M, et al.The creative brain: investigation of brain activity during creative problem solving by Means of EEG and FMRI. Hum Brain Mapp 2009;30:734-48. http://dx.doi.org/10.1002/hbm.20538 